\title{
Why I am not a QBist
}

\author{
Louis Marchildon
}

Received: 13 November 2014 / Accepted: 2 February 2015 / Published online: 18 February 2015

C) The Author(s) 2015. This article is published with open access at Springerlink.com

\begin{abstract}
Quantum Bayesianism, or QBism, is a recent development of the epistemic view of quantum states, according to which the state vector represents knowledge about a quantum system, rather than the true state of the system. QBism explicitly adopts the subjective view of probability, wherein probability assignments express an agent's personal degrees of belief about an event. QBists claim that most if not all conceptual problems of quantum mechanics vanish if we simply take a proper epistemic and probabilistic perspective. Although this judgement is largely subjective and logically consistent, I explain why I do not share it.
\end{abstract}

Keywords Quantum mechanics · Interpretation · Epistemic view ·

Quantum Bayesianism

\section{Introduction}

Ever since its formulation 90 years ago, quantum mechanics has given rise to a lively debate about its interpretation. Many view the questions of measurement and locality as major unsolved, or at best partly and unsatisfactorily solved, problems. Others maintain that measurement and locality are only pseudoproblems, that are eliminated by a proper way of formulating the language or viewing the scope of quantum mechanics. On the whole, the latter view has been maintained, in particular, by Bohr and other adherents of the Copenhagen interpretation.

An important feature of the Copenhagen interpretation is its instrumentalism, or pragmatism [1]. To quote Bohr just once on this [2]: "[I]n our description of nature the

\footnotetext{
L. Marchildon $(\varangle)$

Département de chimie, biochimie et physique, Université du Québec,

Trois-Rivières, QC G9A 5H7, Canada

e-mail: louis.marchildon@uqtr.ca
} 
purpose is not to disclose the real essence of the phenomena but only to track down, so far as it is possible, relations between the manifold aspects of our experience." Heisenberg argued [3] that the "probability function represents a mixture of two things, partly a fact and partly our knowledge of a fact [...] In ideal [pure] cases the subjective element in the probability function may be practically negligible as compared with the objective one." Peierls [4] went further and interpreted the wave function entirely in terms of knowledge, an approach known as the epistemic view of quantum states.

The epistemic view has gained momentum with the development of quantum information theory [5]. At the same time, two new arguments have been raised against it. In my view, both are interesting but neither is decisive. The first one has to do with protective measurements [6]. These measurements allow reconstructing a system's state vector to arbitrary accuracy, with arbitrarily small disturbance. This may suggest that the state vector is real. To be performed, however, protective measurements require some previous knowledge of the state vector, for instance that it is an eigenstate of some specific operator.

The second argument against the epistemic view [7] assumes that (i) a quantum system has a real physical state (parametrized by $\lambda$ ) and that (ii) systems prepared independently have independent physical states. If $\lambda$ uniquely determines the state vector, the latter is ontic. Otherwise, it is epistemic. The argument then proves that epistemic state vectors are inconsistent with predictions of quantum mechanics. It has later been shown [8], however, that the argument fails if a weaker form of assumption (ii) is used.

The epistemic view of quantum states naturally fits in with the subjective interpretation of probability. This has led a number of investigators to develop the approach of Quantum Bayesianism, or QBism, neatly summarized in a recent paper by Fuchs, Mermin and Schack [9]. I believe that QBism is the clearest formulation so far of the epistemic view of quantum states.

I have explained before why I do not uphold the epistemic view of quantum states $[10,11]$. In this paper I intend to sharpen the argument and aim it more specifically at QBism. ${ }^{1}$ After a brief summary of QBism in Sect. 2, I shall make a parallel between it and two related and controversial approaches to knowledge. Section 4 will examine how objections to these approaches also apply to QBism. Additional comments and comparisons will be proposed in Sect. 5.

\section{QBism in a Nutshell}

Quantum Bayesianism, or QBism for short, views "quantum mechanics [as] a tool anyone can use to evaluate, on the basis of one's past experience, one's probabilistic expectations for one's subsequent experience" [9]. QBism explicitly adopts the subjective view of probability, wherein probability assignments express an agent's personal

\footnotetext{
1 Jaeger [12] and Mohrhoff [13] criticize QBism on different grounds. Jaeger believes that "[quantum information's] triumphs are merely technological; they don't in themselves provide direct insight into the physics on which they are based." Mohrhoff argues, from a Kantian and Bohrian perspective, that physics is not about subjective experiences, but about the objectifiable aspects of our experience. Of course my view of QBism does not constitute a criticism of general Bayesian statistics and their wide-ranging applications.
} 
degrees of belief about an event. On the basis of their own specific experience, two agents can legitimately assign different probabilities to the same event.

According to QBism, an agent (say Alice) can use quantum mechanics to model any physical system external to herself. This means that on the basis of her beliefs, Alice assigns a state vector (or density operator) to the system and, through the use of Born's rule, computes probabilities of outcomes resulting from her interaction with the system. Once a specific outcome has occurred, Alice's state vector is correspondingly updated. The system can include anything external to Alice, including other agents. In QBism, "quantum mechanics itself does not deal directly with the objective world; it deals with the experiences of that objective world that belong to whatever particular agent is making use of the quantum theory" [9].

QBism deals quite straightforwardly with alleged conceptual problems of quantum mechanics. In the case of measurement, for instance, one can ask how can a quantum system's state vector suddenly change upon measurement of a dynamical variable, in violation of the continuous and unitary Schrödinger equation? QBists answer that the state vector does not describe the quantum system under investigation, but an agent's beliefs about that system. The "collapse of the state vector" simply reflects the acquisition of new beliefs by the agent.

The problem of nonlocality also stems (in part at least) from collapse, when the latter is viewed as affecting the objective state of a physical system. If Alice and Bob share a pair of particles in the singlet state, Alice's measurement of the spin of her particle appears to instantaneously collapse the state vector of Bob's particle. But for QBists, Alice's measurement simply updates her beliefs and has no effect on Bob's particle. Bob can certainly measure the spin of his particle and, sure enough, proper correlation of results will show up if Alice and Bob subsequently meet and exchange information. In the words of [9],

QBist quantum mechanics is local because its entire purpose is to enable any single agent to organize her own degrees of belief about the contents of her own personal experience. No agent can move faster than light: the space-time trajectory of any agent is necessarily timelike. Her personal experience takes place along that trajectory.

I believe that once the notion of "agent" is precisely defined (for instance, a mentally sane Homo sapiens), QBism is a consistent and well-defined theory. Although QBists generally do not deny the existence of an objective world outside the mind of agents, their resolution of the measurement and nonlocality problems crucially depends on not attributing certain objective properties to the outside world or, at least, on considering such properties as beyond the scope of physical science. This has much in commmon with other instrumentalistic approaches to knowledge, two examples of which I now briefly describe.

\section{Two Related Views}

The first approach to which QBism is related is idealistic philosophy. Going back to George Berkeley and earlier, idealism holds that only mind exists, and that matter is 
an illusion. An extreme form of idealism is solipsism, according to which only one mind exists. The solipsist believes that there is nothing external to his own mind.

There are at least two reasons why solipsism may be attractive. The first one, as Descartes has shown in his Meditations, is that the existence of my own mind is the only thing I can be sure of (except for logico-mathematical statements). Everything else can be subject to doubt. The second reason why solipsism is attractive, which also applies to idealism in general, is that it addresses and solves one of the most profound philosophical questions, the mind-body problem. The dualist's enigma of the relationship between mind and matter, or the materialist's problem of how mind can originate from matter are simply dissolved in the nonexistence of matter.

Of course QBism is neither solipsism nor idealism. It does not altogether deny the existence of matter. But it does share an important methodological rule with idealistic philosoply: the only purpose of science is to organize an agent's (or a mind's) private experience. For idealists, science does not describe matter in an outside world, it organizes the experience of mind (or minds). For QBists, quantum mechanics does not describe electrons, photons or other quantum systems (hereafter collectively referred to as "quantum particles"). It is a tool for agents to make probabilistic statements about their own future experience. Idealism solves the mind-body problem by denying the existence of matter. QBism solves the measurement problem by considering the state of quantum particles as nonexistent or beyond the scope of science.

There is a further analogy between idealism and QBism. For idealists, postulating the existence of matter makes no difference whatsoever to the mind's private experience. Matter is therefore regarded as superfluous and discarded on the basis of Ockham's razor. For QBists, postulating true states for quantum particles makes no difference on an agent's beliefs and the probabilistic predictions he or she makes on that basis. Quantum particle states are therefore considered as superfluous.

The second approach to which QBism is related is behaviorist psychology. Behaviorism claims that psychology should study the observable behavior of humans and animals, without introducing or using the concept of mental states. One of the objectives of psychology is then to predict the response of humans or animals to various kinds of stimuli. Knowledge gained in such investigations can have therapeutic applications. For instance, applying specific stimuli can result in alteration of unwanted behavior like phobias, addictions, etc.

In eschewing the concept of mental states, behaviorists can make relevant predictions without having to consider the difficult question of the relationship between brain and mind. This is like QBists who make predictions and develop optimal betting strategies without attributing states to quantum particles. Mental states in behaviorism correspond to quantum particle states in QBism. However, the analogy is not perfect. Behaviorists in general don't deny the existence of mental states. They just claim that they are irrelevant to psychology (while perhaps being relevant to something else). QBists, however, do in general deny the existence of quantum particle states, or at least their relevance to anything significant.

Overlooking mental states can lead to weird consequences. A few decades ago, some sociologists of science introduced what they called the strong program, which essentially equated science with any ideology or set of beliefs held by a given social group. Advocates of the strong program would study the behavior of scientists in a 
laboratory just like primatologists study the behavior of apes in their natural environment. They would claim to make sense of the scientists' practices without any reference to the objectives or purposes that scientists have when conducting their experiments. The strong program has by now been discredited [14], but it illustrates excesses to which a strictly phenomenological attitude can lead.

\section{Discussion}

In this section, I examine why someone would not adopt each of the two approaches summarized above, and see if the reasons also apply to QBism.

Let us begin with behaviorist psychology. One reason to reject it as a fundamental approach may have to do with personal preferences. An investigator may recognize the effectiveness of behaviorism in treating a number of disorders but, influenced by the perception of his own mental states and their subjective importance, feel that this approach does not provide satisfactory psychological knowledge. But in addition to this personal or subjective reason, there is also an empirical one. No psychologist will deny that there are mental states. Behaviorists do maintain that there is much to do in practical and theoretical psychology without referring to mental states. But clearly, introspection helps to gain at least some insights that the study of stimuli and responses alone cannot get. There are empirical differences between predictions made on the basis of stimuli and responses only, and on the basis of introspection.

How does this translate to QBism? I will come back later to the question of personal preferences, focussing at this stage on empirical differences. As pointed out earlier, there are no empirical differences between the probabilistic predictions made by a QBist and the predictions made by someone who claims that the state vector represents the true state of a quantum particle. The empirical objection to behaviorism therefore doesn't seem to apply to QBism.

This conclusion, however, rests on a far-reaching hypothesis. It will hold if quantum mechanics (or a suitable generalization to relativistic fields, strings and the like) is the ultimate theory of nature. This may be true, but it should certainly be challenged, both on the experimental and theoretical sides. One theoretical challenge specifically consists in attributing real states to quantum particles, e.g. hidden variables as in Bohmian mechanics [15]. Although Bohm's original approach yields predictions in agreement with standard quantum mechanics, straightforward modifications of it predict empirical differences that can be put to the test [16].

Let us now turn to idealist philosophy. There is no doubt that idealism and solipsism are logically consistent and (at least for solipsism) conceptually simple views of the world. Moreover, there is no experiment that can distinguish between idealism and a realistic view of matter. Yet very few people, at least among scientists, are true idealists, let alone solipsists. Why is that?

I can see several reasons why most people are not idealists. The first one is that our intuitive feeling for reality is too strong. We just find it incredible that tables and chairs are not solid matter. The second one has to do with the "order" that we perceive in the phenomena. Again, we find it unbelievable that this order should be due to something solely in the mind. A third reason doesn't apply to idealism in general, but to solipsism 
only. Even if there is nothing outside mind, we do not believe that our experience of other minds functioning, as it were, much like our own, could only be an artefact of our own unique mind.

It is important to point out that these reasons adduced against idealism and solipsism have nothing to do with logical requirements or the results of experiments. In the end, they boil down to personal preferences. The only objection I know to idealism and solipsism is, ultimately, "I don't like it." Such judgement is based on methodological or meta-empirical preferences, rather than on logic and experiments. My only way to convince idealists or solipsists to change their views is to bring them to share my personal preferences.

How can an argument resting on personal preferences eventually move a QBist? As pointed out earlier, most QBists do not deny the existence of quantum particles (i.e. electrons, photons, etc.). They deny that quantum particles have states, or that these states should be the object of science. I will address this group in the first place.

Suppose one believes in the existence of quantum particles. Then one can ask, "How can quantum particles be for quantum mechanics to be true?" Although some may claim that this question has no empirical meaning, it is hard to see how one could maintain that it has no logical meaning. I will now argue that in addition to having meaning, the question is also relevant.

More specifically, I can see three broad types of answers to the above question. I claim that all three are interesting and relevant, even to QBists.

The first possible answer to the question could be that there is a simple, coherent and intuitively appealing way to describe quantum particles that precisely yields the quantum formalism. This is what happens in classical mechanics, where the identification of $m, \mathbf{r}$ and $\mathbf{v}$ with the mass, position and velocity of particles constitutes a noncontroversial way to interpret the formalism. Unfortunately, close to a century of research in quantum foundations has not produced such a simple interpretation. This, however, is no proof that none can be adduced, and research in that direction may still be worthwhile.

The second possible answer could be that there is no way that quantum particles can behave for quantum mechanics to be true. If that were the case, I claim that anyone believing in the existence of quantum particles would have to conclude that quantum mechanics, in spite of its empirical success, cannot be a satisfactory theory of nature.

Fortunately, this answer is not the correct one (at least in nonrelativistic quantum mechanics), since there are counterexamples. The simplest one is probably Bohmian mechanics which, irrespective of personal preferences, provides a clear and consistent way to describe fundamental particles in full agreement with quantum mechanics. Other counterexamples are many-world theories [17], transactional approaches [18] or consistent histories [19].

That brings us to the third possible answer to the question raised above. As I have just outlined, there are many ways the world of quantum particles can be for quantum mechanics to be true. But none has (for many people at least) the cogency that the standard interpretation of classical mechanics has in terms of masses, positions and velocities of particles.

The fact that none of these answers is appealing to QBists leads them to do away with these approaches and stick to the experience of agents. But for anyone who 
believes in the existence of quantum particles, that situation is problematic. How can one be comfortable with entities whose only known ways to behave are unbelievable? Doesn't this lead to look for other avenues and seek new solutions to the problem? ${ }^{2}$ If any known way by which the quantum mechanics of particles can be true raises problems, then these problems ipso facto transfer to QBism.

\section{Concluding Remarks}

Other attempts that broadly fall within the epistemic view of quantum states have been proposed in recent years to deal with the conceptual problems of quantum mechanics. I shall briefly mention two which, although not explicitly referring to QBism, bear relations to it.

The first one was proposed by Ulfbeck and Bohr and is called genuine fortuitousness [21]. In the view of these investigators, there are just no quantum particles. A Geiger counter, detecting what we would normally call the decay products of a radioactive atom, clicks according to well-defined probabilistic rules but without any cause. Quantum mechanics is a theory that predicts such probabilities, in the context of specific arrangements of preparation and measurement devices, specified exclusively in classical terms. This approach can be viewed as a radical version of QBism, where even the existence of quantum particles is denied. I have criticized it elsewhere [11]. The criticism essentially boils down to asking how can preparation and measurement devices be ultimately made of constituents that have no existence?

The second, more recent approach, is due to Englert [22]. The way it deals with measurement and nonlocality is very close to QBism, but it doesn't have the sharpness of the latter. It uses for instance the ill-defined concept of event, which involves quantum particles ("the emission of a photon by an atom") but is also assumed to be irreversible. But elementary quantum processes, governed by unitary evolution, are never irreversible. Even practical irreversibility essentially requires the specification of a classical context, for instance the distinction between emission in a vacuum (practically irreversible) and emission in a cavity (reversible).

QBism shares with the statistical interpretation of quantum mechanics, advocated for instance by Ballentine [23], the idea that the state vector is not an objective attribute of an individual system. Ballentine, however, rejects the view that the state vector represents knowledge, and considers the state vector as an objective description of an ensemble of systems. He also quite definitely commits to ontic properties of individual systems (like definite particle positions, p. 361), a commitment foreign to QBism.

I should finally mention a recent paper by Mermin [24], who uses ideas drawn from QBism to examine the difficult (and purely classical) problem of the moving Now. How can one reconcile the subjective perception of a moving present with the four-dimensional block world view of space-time? Mermin's answer is to take the experience of agents as the prime object of science, and to argue that space-time has no objective reality. He then shows how to connect the individual experience of Now with features of space-time diagrams, and explains that the Nows of two different

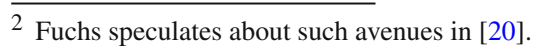


agents will always agree when they meet. But that does not settle the issue, for the really deep question is the following: How can the constituents of the agent (atoms, molecules or cells), which have no Now experience, behave so that their aggregate has a Now experience? This problem may not be solved for quite a while, but in my view it is definitely a problem of science.

Acknowledgments I am grateful to the Natural Sciences and Engineering Research Council of Canada for financial support. I thank Chris Fuchs and Ruth Kastner for useful comments.

Open Access This article is distributed under the terms of the Creative Commons Attribution License which permits any use, distribution, and reproduction in any medium, provided the original author(s) and the source are credited.

\section{References}

1. Stapp, H.P.: The Copenhagen interpretation. Am. J. Phys. 40, 1098-1116 (1972)

2. Bohr, N.: Atomic Theory and the Description of Nature, p. 18. Ox Bow Press, Woodbridge (1987)

3. Heisenberg, W.: Physics and Philosophy. The Revolution in Modern Science, pp. 19-27. Harper, New York (2007)

4. Peierls, R.: In defence of 'measurement'. Phys. World 4(1), 19-20 (1991)

5. Fuchs, C.A., Peres, A.: Quantum theory needs no 'interpretation'. Phys. Today 53(3), 70-71 (2000)

6. Aharonov, Y., Anandan, J., Vaidman, L.: Meaning of the wave function. Phys. Rev. A 47, 4616-4626 (1993)

7. Pusey, M.F., Barrett, J., Rudolph, T.: On the reality of the quantum state. Nature Phys. 8, 475-478 (2012)

8. Emerson, J., Serbin, D., Sutherland, C., Veitch, V.: The whole is greater than the sum of the parts: on the possibility of purely statistical interpretations of quantum theory. Preprint arXiv:1312.1345 (2013)

9. Fuchs, C.A., Mermin, N.D., Schack, R.: An introduction to QBism with an application to the locality of quantum mechanics. Preprint arXiv:1311.5253 (2013)

10. Marchildon, L.: Why should we interpret quantum mechanics? Found. Phys. 34, 1453-1466 (2004)

11. Marchildon, L.: Quantum mechanics needs interpretation. Int. J. Adv. Syst. Meas. 2, 131-141 (2009)

12. Jaeger, G.: Entanglement, Information, and the Interpretation of Quantum Mechanics, Sect. 3.7. Springer, Berlin (2009)

13. Mohrhoff, U.: QBism: a critical appraisal. Preprint arXiv:1409.3312 (2014)

14. Bunge, M.: A critical examination of the new sociology of science (I and II). Philos. Soc. Sci. 21, 524-560 (1991). 22, 46-76 (1992)

15. Bohm, D.: A suggested interpretation of the quantum theory in terms of 'hidden' variables (I and II). Phys. Rev. 85, 166-193 (1952)

16. Valentini, A.: De Broglie-Bohm pilot-wave theory: many worlds in denial? In Many Worlds? Everett, Quantum Theory, and Reality, pp. 476-509. Oxford University Press, Oxford (2010)

17. Saunders, S., Barrett, J., Kent, A., Wallace, D. (eds.): Many Worlds? Everett, Quantum Theory, and Reality. Oxford University Press, Oxford (2010)

18. Kastner, R.E.: The Transactional Interpretation of Quantum Mechanics. The Reality of Possibility. Cambridge University Press, Cambridge (2013)

19. Griffiths, R.B.: Consistent Quantum Theory. Cambridge University Press, Cambridge (2002)

20. Fuchs, C.A.: QBism, the perimeter of quantum Bayesianism. Preprint arXiv:1003.5209 (2010)

21. Ulfbeck, O., Bohr, A.: Genuine fortuitousness. Where did that click come from? Found. Phys. 31, 757-774 (2001)

22. Englert, B.G.: On quantum theory. Preprint arXiv:1308.5290 (2013)

23. Ballentine, L.E.: The statistical interpretation of quantum mechanics. Rev. Mod. Phys. 42, 358-381 (1970)

24. Mermin, N.D.: QBism as CBism: solving the problem of 'the Now'. Preprint arXiv:1312.7825 (2013) 